\title{
Suicide gene-armed measles vaccine virus for the treatment of AML
}

\author{
STEFANIE MAURER ${ }^{1,2}$, HELMUT R. SALIH ${ }^{1-3}$, IRINA SMIRNOW ${ }^{4,5}$, \\ ULRICH M. LAUER ${ }^{4,5}$ and SUSANNE BERCHTOLD ${ }^{4,5}$
}

\author{
${ }^{1}$ Clinical Collaboration Unit Translational Immunology, German Cancer Consortium (DKTK) and \\ German Cancer Research Center (DKFZ), Partner site Tuebingen; ${ }^{2}$ DFG Cluster of Excellence 2180 'Image-guided \\ and Functional Instructed Tumor Therapy' (IFIT); ${ }^{3}$ Department of Hematology and Oncology, \\ Eberhard Karls University; ${ }^{4}$ Department of Internal Medicine VIII, University Hospital Tuebingen; \\ ${ }^{5}$ German Cancer Consortium (DKTK), DKFZ partner site Tuebingen, D-72076 Tuebingen, Germany
}

Received January 3, 2019; Accepted May 13, 2019

DOI: 10.3892/ijo.2019.4835

\begin{abstract}
Virotherapy comprises a novel therapeutic approach to selectively eliminate cancer cells. Preclinical, as well as clinical data have demonstrated the efficacy of tumor-selective (oncolytic) viruses in hematological malignancies. In this study, we infected AML cell lines and primary AML cells from patients with measles vaccine virus either expressing GFP or armed with super cytosine deaminase, which converts the prodrug, 5-fluorocytosine, into the chemotherapeutic compound, 5-fluorouracil. Target cell density of the measles entry receptor, CD46, infection rates of targeted leukemic cells, tumor cell viability, and apoptotic rates were determined. We found that measles vaccine virus infected the leukemic blasts and profoundly diminished the number and viability of leukemic cells via the induction of apoptosis. The conversion of 5-fluorocytosine to 5-fluorouracil exerted a potent additive tumoricidal effect. This was also observed in cases when leukemic cells displayed only moderate susceptibility to the oncolytic virus and hence direct oncolysis. Taken together, in this study, we provide a first characterization of the combinatorial use of measles vaccine virus and 5-fluorouracil for treatment of AML. Our approach to site-specifically
\end{abstract}

Correspondence to: Professor Ulrich M. Lauer, Department of Internal Medicine VIII, University Hospital Tuebingen, Otfried-Mueller-Str. 10, D-72076 Tuebingen, Germany

E-mail: ulrich.lauer@uni-tuebingen.de

Abbreviations: AML, acute myeloid leukemia; dpi, days post infection; ELISA, enzyme-linked immunosorbent assay; 5-FC, 5-fluorocytosine; 5-FU, 5-fluorouracil; HD, healthy donor; IFIT, interferon-induced protein with tetratricopeptide repeats; IFN, interferons; $\mathrm{MeV}$, measles vaccine virus; MOI, multiplicity of infection; SCD, super cytosine deaminase; TMRE, tetramethylrhodamine ethyl ester; UPN, unique patient number

Key words: oncolytic virus, measles vaccine virus, virotherapy, acute myeloid leukemia, chemotherapy, prodrug produce the active drug and combine this agent with the direct lytic effect of virotherapy may overcome present limitations and constitutes a feasible method with which to introduce 5-fluorouracil in the treatment of AML.

\section{Introduction}

The response to the induction of chemotherapy is of major prognostic relevance for the outcome of patients with acute myeloid leukemia (AML). In light of the tolerable toxicity, the achievable drug dosage constitutes a limiting factor for therapeutic success (1). Clinical observations of leukemia regression coinciding with virus infections served as an impetus to evaluate the use of oncolytic viruses as a novel therapeutic strategy (2), particularly since viruses have been found to specifically mediate the lysis of tumor cells, whereas non-malignant cells are spared (3). Over the recent years, oncolytic virotherapy has been investigated for the treatment of various malignant diseases in numerous clinical trials (4); however, success thus far is limited since tumor cells may display resistance to virus-induced lysis. To enhance the efficacy of oncolysis, several strategies have been employed. Among others, oncolytic viruses have been engineered to express suicide genes which facilitate the conversion of inactive prodrugs into toxic agents, resulting in targeted, i.e., tumor cell-restricted, chemotherapy (5). Measles vaccine virus $(\mathrm{MeV})$ has been shown to possess oncolytic potential in a variety of tumors and has been evaluated in several recruiting clinical trials, including multiple myeloma, mesothelioma and ovarian cancer (6). Interim reports show substantial improvement for patients with several malignancies and attribute an excellent safety record, which is in line with the well-known safety profile of $\mathrm{MeV}$ applied as vaccine for a number of decades. However, thus far, there are no trials studies on the oncolytic effects of $\mathrm{MeV}$ in the treatment of AML (https://www.clinicaltrials.gov/).

Upon viral infection within the host cell, pathogen-associated molecular patterns are generated, which in turn are sensed by cytoplasmic receptors, such as retinoid acid inducible gene I (RIG-I) or melanoma differentiation-associated 
gene 5 (MDA5) $(7,8)$. The activation of the latter induces the production and secretion of type I interferons (IFNs) (7), thereby facilitating the activation of the Janus kinase/signal transducer and activator of transcription (JAK/STAT) signaling cascade (9). IFN-stimulated genes are inducers of an antiviral state of infected, as well as neighboring cells to prevent viral spread (10). Yet, viral mechanisms with which to evade immunosurveillance have co-evolved, e.g., wild-type measles virus interferes with the aforementioned induction of IFN secretion (11).

In this study, a virotherapeutic $\mathrm{MeV}$ armed with super cytosine deaminase (MeV-SCD), a fusion protein consisting of yeast cytosine deaminase and yeast uracil phosphoribosyl transferase $(3,12)$, was preclinically characterized for the treatment of AML. SCD catalyzes conversion of the inactive prodrug 5-fluorocytosine (5-FC) into the therapeutically active and clinically approved compound 5-fluorouracil (5-FU) (13). 5-FU mediates its effects by the inhibition of thymidylate synthase and incorporation of its metabolites into RNA and DNA (14). Due to the heterogeneity associated with hematological malignancies and particularly AML, the combined application of therapeutic agents is a strategy widely practiced in clinical hematology $(1,15)$.

We here evaluated the combinatorial use of vaccine-derived MeV-SCD and locally generated 5-FU for the treatment of AML cell lines, as well as primary AML cells from patients. While $\mathrm{MeV}$ displayed direct cytotoxicity in the absence of the prodrug, the conversion of 5-FC to the active compound, 5-FU, exerted a potent additive anti-tumor effect. Notably, this was also observed when the leukemic cells displayed only moderate susceptibility to the oncolytic virus and the respective direct oncolysis. Our approach to tumor-selectively produce active 5-FU and to combine this agent with the direct lytic effect of virotherapy may overcome the limitations of systemic chemotherapy dosage (1) and constitutes a feasible method with which to introduce 5-FU into the treatment of AML.

\section{Materials and methods}

Cells and cell culture. Peripheral blood mononuclear cells (PBMCs) cells of patients at the time of diagnosis and PBMCs or bone marrow (BM) of healthy donors were isolated by Ficoll/Biocoll (Biochrom AG) density gradient centrifugation. The clinical characteristics of the individuals included in this study are presented in Tables I and II. This study was approved by the Ethics Committee at the Medical Faculty of the Eberhard Karls University and the University Hospital Tuebingen (reference no. 13/2007V). Informed consent was obtained from all patients and healthy donors in accordance with the Helsinki protocol. Human AML cell lines MONO-MAC-6 (MM-6), NOMO-1 and SKM-1 were obtained from the German Collection of Microorganisms and Cell Cultures. Authenticity was determined by validating the respective immunophenotype described by the provider using FACS every 6 months and specifically prior to use in the experiments. The MM-6 cells were cultured in MEM alpha medium (Life Technologies) supplemented with $1 \%$ penicillin/streptomycin (Lonza) and 10\% FCS (Biochrom). The NOMO-1 and SKM-1 cells were cultured in RPMI-1640 medium (Life Technologies) supplemented with $1 \%$ penicillin/streptomycin and $10 \%$ (NOMO-1) or $20 \%$ (SKM-1) FCS. Primary blood cells were viably frozen in RPMI-1640 supplemented with 20\% FCS and $10 \%$ dimethyl sulfoxide (Sigma-Aldrich), freshly thawed for each experiment and cultured in RPMI-1640 medium supplemented with $10 \%$ human AB serum (c.c.pro $\mathrm{GmbH}$ ). Vero cells were obtained from the German Collection of Microorganisms and Cell Cultures and cultured in DMEM medium (Sigma-Aldrich) supplemented with $10 \%$ FCS. The cells were kept in a humidified incubator at $37^{\circ} \mathrm{C}$, containing $5 \% \mathrm{CO}_{2}$.

Propagation and titration of measles vaccine virus. The generation of measles vaccine viruses MeV-GFP (encoding for GFP) and MeV-SCD (encoding for suicide gene SCD) was conducted as previously described (3). In brief, virus stocks were produced in Vero cells. A total of $1 \times 10^{7}$ Vero cells were seeded in $15-\mathrm{cm}$ plates, washed once after $24 \mathrm{~h}$ with phosphate-buffered saline (Sigma Aldrich), and infected for $3 \mathrm{~h}$ at an MOI of 0.03 in Opti-MEM (Invitrogen; Thermo Fisher Scientific). Subsequently, the inoculum was removed and DMEM containing 10\% FCS was added. After microscopy had assured maximum infection rates [circa $54 \mathrm{~h}$ post-infection (hpi)], the medium was removed and the Vero cells were scraped into $1 \mathrm{ml}$ Opti-MEM. The release of the virus was achieved by freeze-thaw lysis. Following centrifugation $\left(1,900 \mathrm{x} \mathrm{g}\right.$, for $15 \mathrm{~min}$ at $\left.4^{\circ} \mathrm{C}\right)$, the supernatants were stored at $-80^{\circ} \mathrm{C}$. Viral titers were assessed by using the tissue culture infective dose 50 endpoint titration according to Spearman and Kärber on Vero cells $(16,17)$.

Infection of cells with measles vaccine virus. For viral infection, the cells were centrifuged for $5 \mathrm{~min}$ at $210 \mathrm{x} \mathrm{g}$ at room temperature and resuspended in Opti-MEM containing distinct amounts of viral particles. MOCK-infected cells were resuspended in Opti-MEM only. The cells were always infected at a multiplicity of infection (MOI) of 10. Following $2 \mathrm{~h}$ of incubation, the cells were centrifuged again for $5 \mathrm{~min}$ at $210 \mathrm{x} g$ at room temperature and then resuspended in culture medium. Where indicated, $5-\mathrm{FC}$ or $5-\mathrm{FU}$ was added at a concentration of $1 \mathrm{mM} 24$ hpi.

Flow cytometry. Flow cytometry was performed using direct fluorescence-conjugates and their respective controls. Phycoerythrin (PE)-labeled anti-human CD46 antibody and PE-labeled IgG1 isotype control were from eBioscience. CD117-PeCy7 and CD33-BV421 conjugates were from BD Biosciences. Dead cells were excluded using either 7-aminoactinomycin D (BioLegend) or using Fixable Aqua (Invitrogen; Thermo Fisher Scientific) following extracellular staining according to the manufacturer's instructions. Cells employed in the virus infection experiments were fixed using cytofix buffer (BD Biosciences). Acquisition was done using a Canto-II (BD Biosciences) or an Attune NxT (Life Technologies) cytometer. Viable, single cells were used for further analysis as depicted in Fig. S1 apart from tetramethylrhodamine ethyl ester (TMRE) staining where only subcellular debris was excluded.

Cell viability assays. Cell viability was determined using the CellTiter-Blue assay. The cells were infected with MeV-GFP 


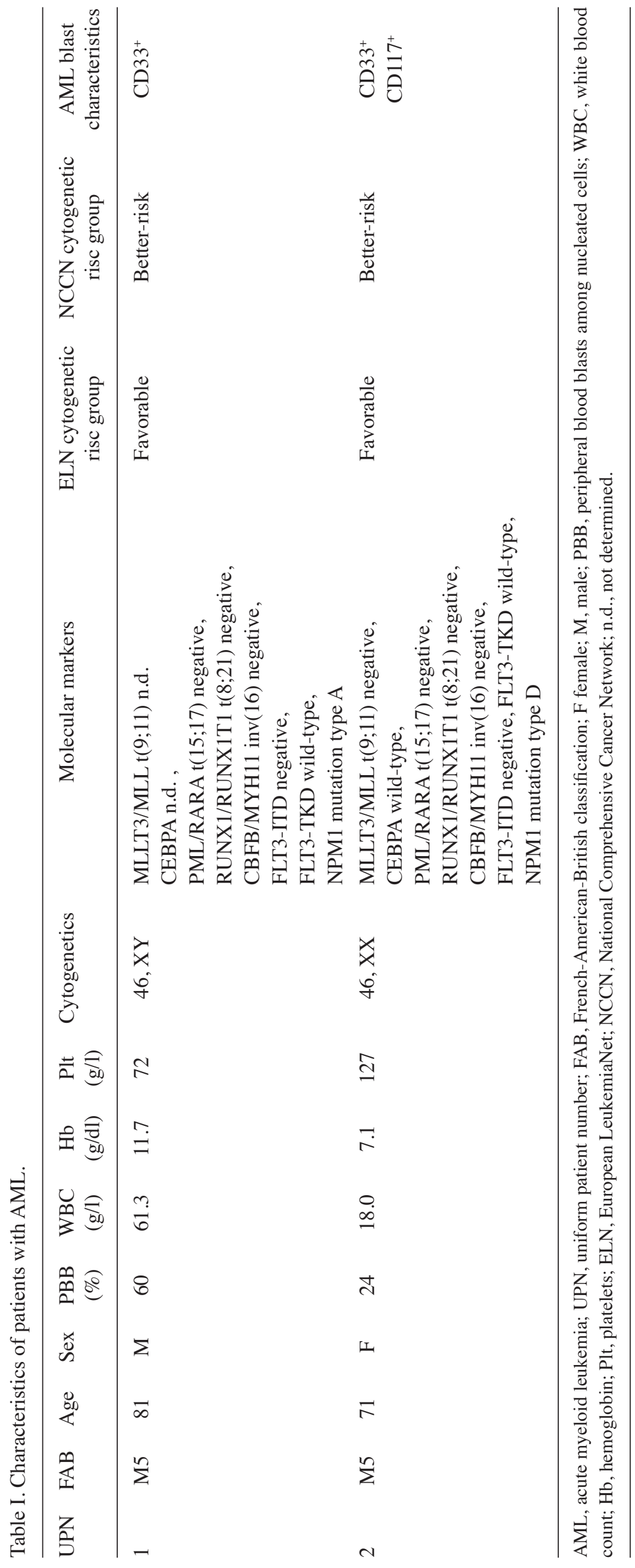


Table II. Characteristics of healthy donors

\begin{tabular}{lcc}
\hline & Count & $\%$ \\
\hline $\begin{array}{l}\text { Total no. of donors } \\
\text { Age (years) }\end{array}$ & 7 & 100 \\
$\quad$ Mean & 49 & \\
Range & $21-77$ & \\
Sex of donors & & \\
Female & 2 & 71 \\
Male & 5 & \\
\hline
\end{tabular}

and MeV-SCD and then seeded in 96-well plates. At 96 hpi, CellTiter-Blue reagent was added and the cells were incubated at $37^{\circ} \mathrm{C}$ for $2 \mathrm{~h}$. Measurements were performed on a microtiter plate reader Tecan Genios Plus (Tecan) using an excitation filter of $584 \mathrm{~nm}$ and Xfluor4 software (Tecan). For combined analysis, results obtained with MOCK infection were set to $100 \%$ in each individual data set. In parallel, cells were stained with Trypan blue and the percentage of dead cells was determined using a Countess II FL Automated Cell Counter (Thermo Fisher Scientific).

Assessment of the mitochondrial transmembrane potential $\triangle \Psi m$ by TMRE staining. The cells were infected with $\mathrm{MeV}-\mathrm{SCD}$ and MeV-GFP and then seeded in 6-well plates. At 96 hpi, the cells were harvested, washed once with PBS, resuspended in $1 \mathrm{ml}$ PBS containing $50 \mathrm{nM}$ TMRE (Molecular Probes) and incubated for $20 \mathrm{~min}$ at $37^{\circ} \mathrm{C}$. Subsequently, $2 \mathrm{ml}$ PBS were added and the cells were centrifuged at $210 \mathrm{x}$ g for $5 \mathrm{~min}$ at room temperature. The cell pellet was resuspended in PBS supplemented with $1 \%$ FCS. Flow cytometric analysis was performed on a FACSCalibur flow cytometer (BD Biosciences) using CellQuest software (BD Biosciences).

Western blot analysis. The cells were infected with MeV-GFP at an MOI of 10 . At the indicated time points $(24,48,72$ or $96 \mathrm{hpi}$ ), the cells were centrifuged at $210 \mathrm{x} \mathrm{g}$ for $5 \mathrm{~min}$ at room temperature, washed once with PBS and lysed in lysis buffer [50 $\mathrm{mM}$ Tris, $150 \mathrm{mM} \mathrm{NaCl}$ and $1 \%$ IGEPAL CA-630 (Sigma-Aldrich)]. Following 3 freeze-thaw cycles, cell debris was removed by centrifugation at $16000 \mathrm{x} \mathrm{g}$ for $10 \mathrm{~min}$ at $4^{\circ} \mathrm{C}$. The protein concentration in the supernatants was determined using the Bradford protein assay (Bio-Rad Laboratories). A total of $50 \mu \mathrm{g}$ of protein were separated by $8 \%$ SDS-PAGE and transferred to hydrophobic polyvinylidene difluoride (PVDF) membranes (Amersham Hybond-P, GE Healthcare). The membranes were blocked in 5\% powdered milk (Carl Roth) in TBS-T (50 mM Tris, $150 \mathrm{mM} \mathrm{NaCl}, 0.02 \%$ Tween-20, $\mathrm{pH}$ 7.4) and then incubated with primary antibodies [anti-phospho-signal transducer and activator of transcription 1 (STAT1), 1:1,000 (Cell Signaling Technology, cat. no. 9167); anti-interferon induced protein with tetratricopeptide repeats (1IFIT1), 1:1,000 (GeneTex, cat. no. GTX 103452); anti-vinculin, 1:5,000 (Sigma-Aldrich, cat. no. V9131)] with gentle shaking overnight at $4^{\circ} \mathrm{C}$. After washing the membranes 3 times with TBS-T, secondary antibodies [horseradish peroxidase-conjugated goat anti-rabbit
(Bio-Rad Laboratories, cat. no. 170-6515, 1:8,000) or goat anti-mouse (Bio-Rad Laboratories, cat. no. 1706516, 1:8,000)] were added for $1 \mathrm{~h}$ at room temperature. The membranes were then again washed 3 times with TBS-T. Proteins were visualized using the ECL Western Blotting Detection Reagent kit (GE Healthcare). Molecular weights were determined using a pre-stained protein ladder (PageRuler Plus, Thermo Fisher Scientific). The expression levels of phospho-STAT1, as well as IFIT-1 were semi-quantitatively analyzed using ImageJ software, normalized to vinculin density.

Enzyme-linked immunosorbent assay (ELISA). The cells were infected with MeV-GFP at an MOI of 10. At 24, 48, 72 and $96 \mathrm{hpi}$, the supernatants were collected and the concentration of IFN- $\beta$ was determined using the VeriKine Human IFN- $\beta$ ELISA kit (PBL Interferon Source) according to the manufacturer's instructions.

Statistical analysis. For statistical analysis, GraphPad Prism 7 software (GraphPad Software, Inc.) was used. Mean values and where indicated SD are shown. The equal distribution of data was tested using the Shapiro-Wilk normality test. The 95\% confidence level was used and P-values were calculated with a two-tailed paired t-test (parametric).

\section{Results}

MeV specifically exerts oncolytic activity against AML cells. As a first step, we investigated whether AML cells are susceptible to $\mathrm{MeV}$ infection. To this end, $3 \mathrm{AML}$ cell lines (MM-6,NOMO-1 and SKM-1) were concurrently infected with the GFP-expressing measles vaccine virus vector $\mathrm{MeV}$-GFP at an MOI of 10 or MOCK-infected. The MM-6, NOMO-1 and SKM-1 cells were found to be susceptible to $\mathrm{MeV}$ infection as determined by flow cytometry (Fig. 1A; gating strategy is displayed in Fig. S1). Time course analyses of $\mathrm{MeV}$ infection revealed highest rates of infection (indicated by percentages of GFP positive cells) at 2 days post-infection (dpi) (Fig. S1). These observations suggest the following: i) Direct oncolytic cell death of infected AML cells; and/or ii) proliferation of primarily infection resistant and therefore, GFP-negative AML cells. Consequently, we assessed rates of direct oncolysis upon viral infection by determining cell viability (Fig. 1B and C). Trypan blue staining, as well as the analysis of viability based on the metabolic activity at $4 \mathrm{dpi}$, revealed that $\mathrm{MeV}$ infection reduced both the percentage of viable cells, as well as the metabolic activity of AML cells as compared to the MOCK controls, albeit to a different extent in the AML cell lines and viability analyses employed. Although there was a clear trend of $\mathrm{MeV}$-mediated oncolysis in all cases, the results obtained with the metabolic activity of the tumor cells did not always reach statistical significance. The observed inter-assay variability may be due to methodological differences with regard to sensitivity and parameters analyzed. This phenomenon was paralleled by the disruption of the mitochondrial membrane potential, as identified by TMRE negativity as a marker of apoptosis in flow cytometric analyses. The MM-6 cells proved to be most susceptible to $\mathrm{MeV}$-mediated oncolysis as shown by a reduction in relative cell viability by $66 \%$ compared to the MOCK control (from 100 to $34 \%$ ) and a 3-fold increase in 
A
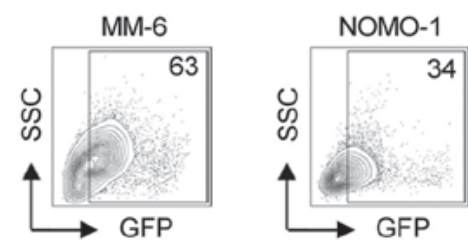

$\mathrm{B}$
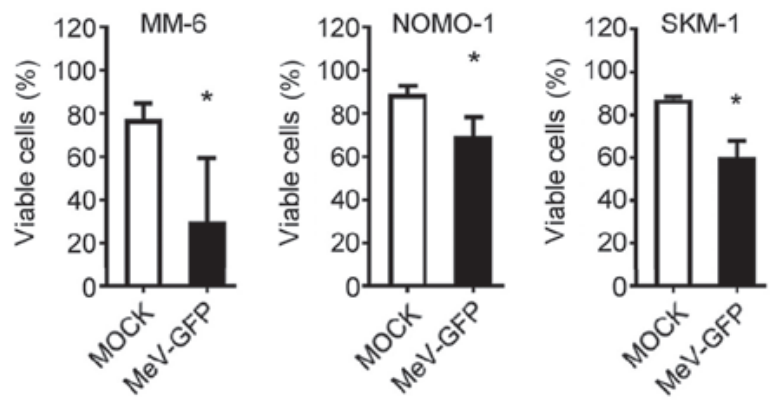

C
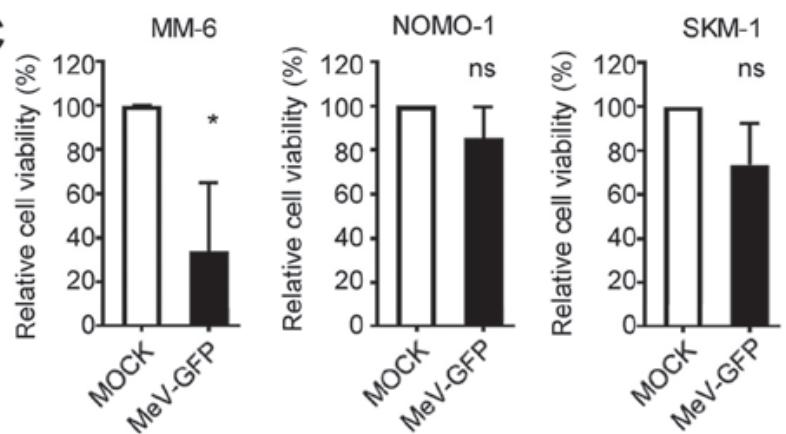

$\mathrm{E}$
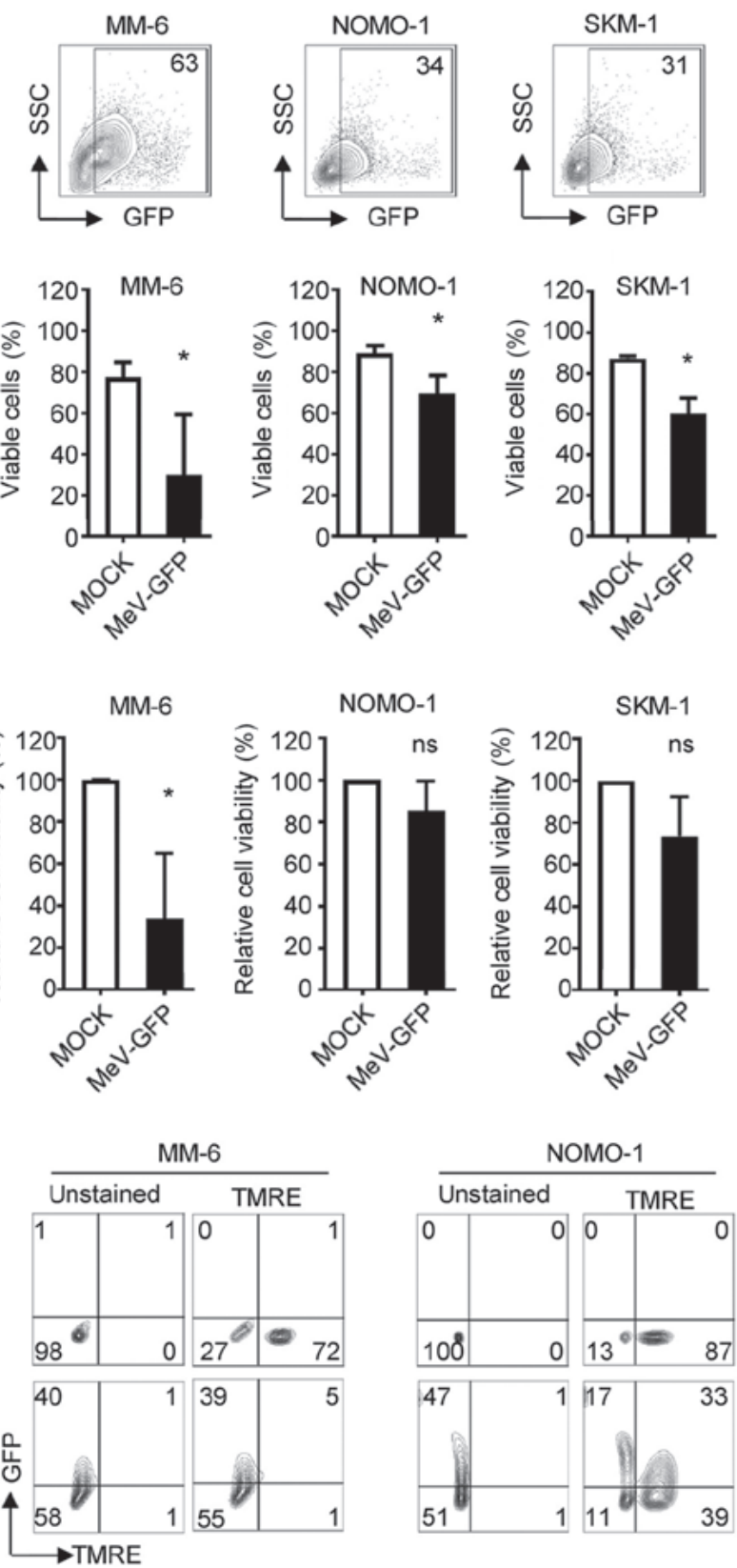

$\mathrm{D}$
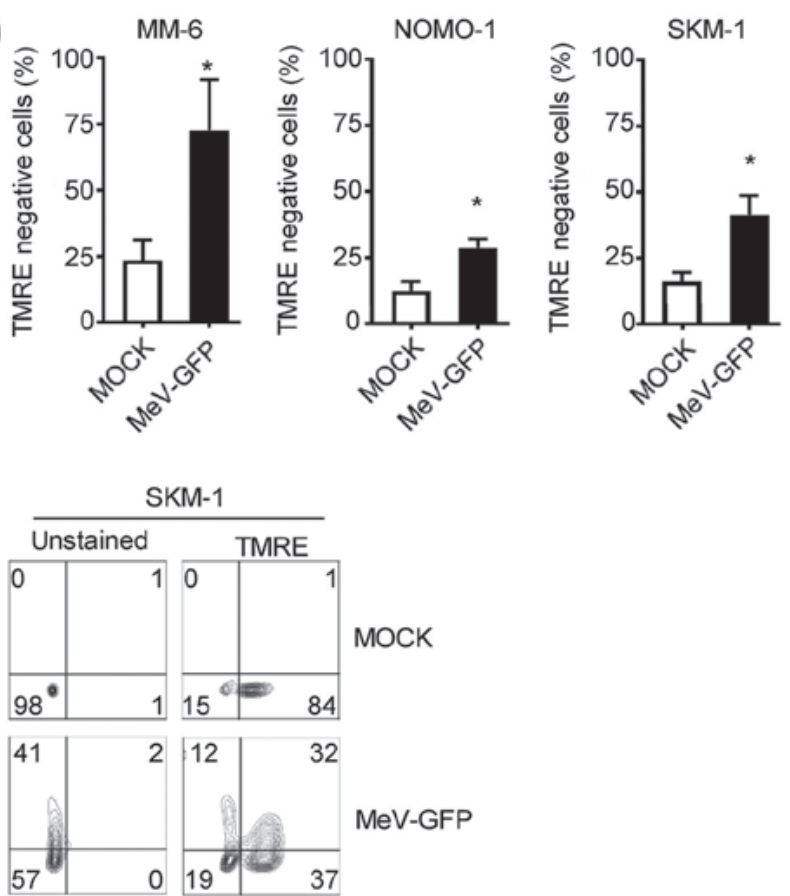

Figure 1. AML cell lines can be infected by MeV. The indicated cells were infected with MeV-GFP or MOCK infected and analyzed 4 dpi. (A) GFP expression of leukemia cells was determined by FACS analysis. The percentage of positive cells is indicated. (B) Cell number was determined using Countess Automated Cell Counter. The percentage of living cells is indicated. (C) Viability of cells was measured using the CellTiter-Blue viability assay. (D and E) Apoptosis was determined by staining with TMRE and subsequent FACS analysis. (D) The percentage of TMRE-negative cells is indicated. (E) Apoptosis rates were assessed with regard to GFP expression. (B-D) Values depict means of data from $n=4$ independent experiments with standard deviation. Statistically significant differences $(\mathrm{P}<0.05)$ are indicated by asterisk ( ()$;$ ns, not significant. AML, acute myeloid leukemia; MeV, measles vaccine virus; TMRE, tetramethylrhodamine ethyl ester.

the percentage of TMRE-negative cells (from 24 to 73\%). The SKM-1 cells also proved to be susceptible to MeV-mediated oncolysis, as shown by a reduction in relative cell viability by $27 \%$ compared to the MOCK control (from 100 to $73 \%$ ) and a 2.6-fold increase in the percentage of TMRE-negative cells (from 16 to 42\%). The NOMO-1 cells were most resistant, as relative viability was merely reduced by $15 \%$ (from 100 to $85 \%$ ) and the proportion of TMRE-negative cells was found to increase 2.4-fold (from 12 to 29\%) upon $\mathrm{MeV}$ infection as compared to the MOCK control (Fig. 1C and D). A substantial fraction of apoptotic cells (MM-6, 39 of 94\% total; NOMO-1, 17 out of $28 \%$ total; and SKM-1, 12 of 31\% total) were found to be infected with MeV-GFP, as indicated by the expression of the virus-encoded marker protein, GFP (Fig. 1E).

Subsequently, we set out to determine whether $\mathrm{MeV}$ can specifically target leukemic cells among the PBMCs of a given patient with AML. Alike AML cell lines, $\mathrm{MeV}$ infection of primary AML cells revealed highest rates of infection (GFP-positive cells) between 2 and 3 days post-infection, as observed by fluorescence microscopy (Fig. S2). Of note, the formation of multinucleated syncytia was not detectable in cells from patients with AML or in the NOMO-1 and SKM-1 cell lines, but only in the MM- 6 cells. FACS analysis revealed that the AML blasts identified according to the individual 
A

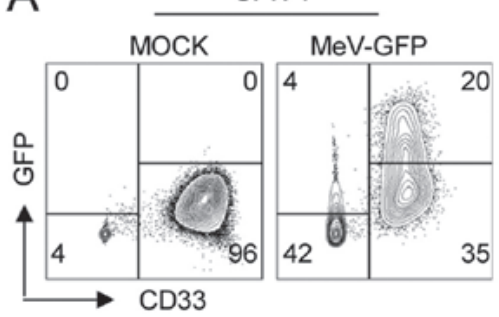

UPN 2

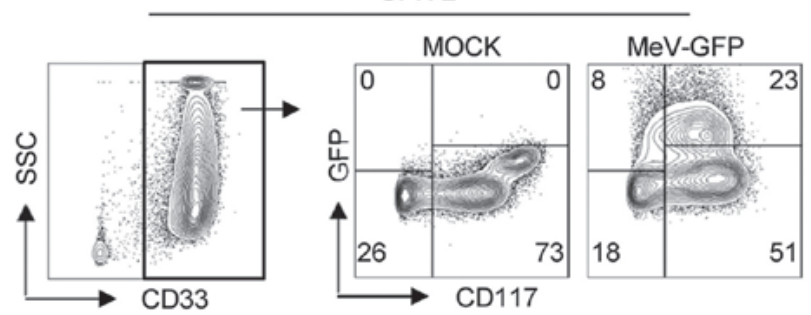

C

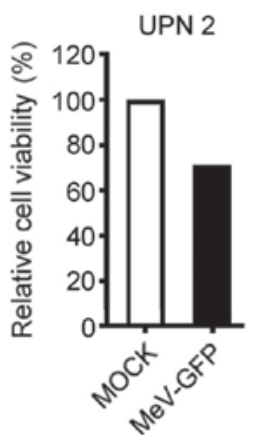

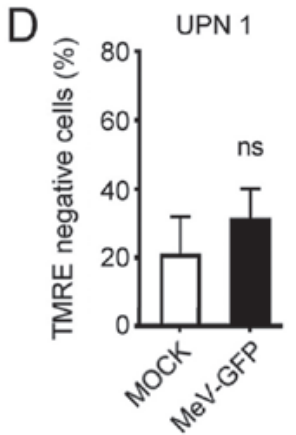
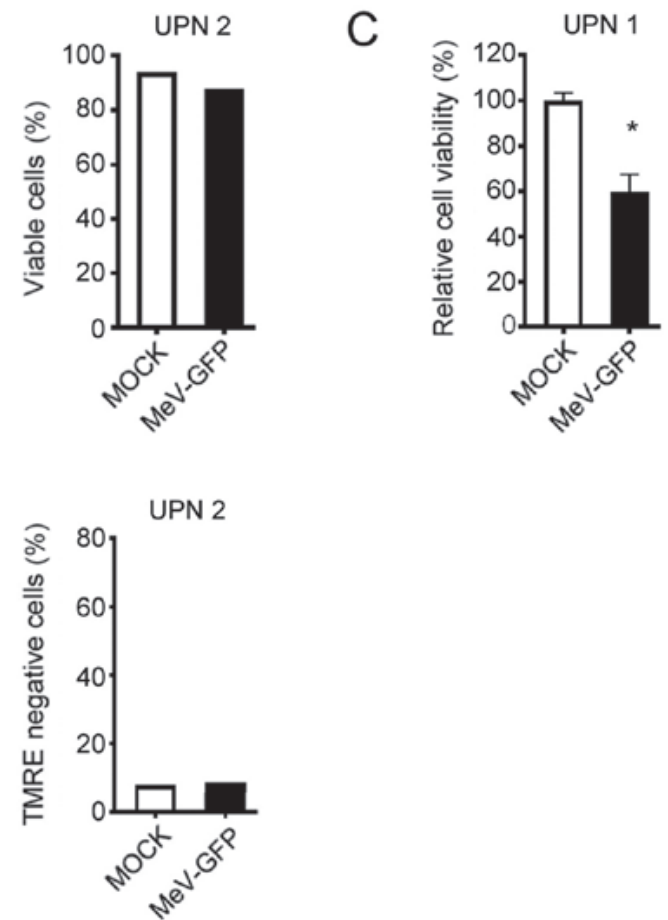

Figure 2. Primary AML cells can be infected with MeV. The PBMC fraction of patients with AML were infected with MeV-GFP or MOCK infected and analyzed at 4 dpi. (A) GFP expression of PBMCs from UPN 1 or UPN 2 was investigated by FACS. The percentage of positive cells is indicated. One representative result out of 3 experiments with similar results is shown. (B) Cell number was determined using the Countess Automated Cell Counter. The percentage of living cells is indicated. UPN $1(n=5)$; UPN $2(n=2)$. (C) Viability of cells was measured using CellTiter-Blue viability assay. UPN 1 (n=5); UPN $2(n=1)$. (D) Apoptosis was determined by staining with TMRE and subsequent FACS analysis. The percentage of TMRE negative cells is indicated. UPN 1 ( $\mathrm{n}=3$ ); UPN 2 (n=1). (B-D) Values depict means of data from the indicated number of independent experiments with standard deviation. Statistically significant differences $(\mathrm{P}<0.05)$ are indicated by asterisk ("); ns, not significant. AML, acute myeloid leukemia; MeV, measles vaccine virus; TMRE, tetramethylrhodamine ethyl ester; UPN, unique patient number; PBMCs, peripheral blood mononuclear cells.

disease characteristics of the respective patients [unique patient number (UPN) $1, \mathrm{CD} 33^{+}$; UPN $\left.2, \mathrm{CD} 33^{+} / \mathrm{CD} 117^{+}\right]$ displayed substantial infection rates at $96 \mathrm{hpi}$ with MeV-GFP at an MOI of 10, whereas non-malignant cells of the respective patients were differentially affected: While CD33- cells from UPN 1 were only slightly affected, benign cells from UPN 2 were also found to express GFP at a low extent (Fig. 2A). With UPN 1, the percentage of viable cells and even more the viability of PBMCs was lowered upon $\mathrm{MeV}$ infection as compared to the MOCK control (Fig. 2B and C). Again, $\mathrm{MeV}$ infection also induced the apoptosis of PBMCs of UPN 1 (32\% compared to $21 \%$ in MOCK-infected cells) (Fig. 2D), while no or only minor alterations were observed with the cells from AML patient UPN 2 regarding the percentage of viable cells and the induction of apoptosis thus displaying a relative resistance to $\mathrm{MeV}$-mediated oncolysis (Fig. 2B-D). Notably, when the PBMCs of healthy donors (HD) were cultured in the presence of MeV-GFP, the infection rates were comparable to those obtained with the PBMCs from patients with AML (Fig. 3A).
However, $\mathrm{MeV}$ infection did not considerably hamper the percentage of viable cells and the metabolic activity of PBMCs obtained from HD (Fig. 3B and C). Moreover, BM cells from $\mathrm{HD}$ were not substantially infected by $\mathrm{MeV}$ and thus, no reduction in cell numbers were observed in this model.

Thus, AML cell lines, as well as patient-derived AML blasts are infectable with MeV-GFP, yet the direct oncolytic efficacy was generally low (apart from the MM-6 cells) with a reduction of cell viability of $<50 \%$.

AML cells express CD46 and initiate IFN signaling upon infection. We then set out to unravel the mechanisms underlying the relative resistance of AML cells to MeV-mediated oncolysis. The MM-6, NOMO-1 and SKM-1 cell lines displayed a substantial surface expression of CD46 (Fig. 4A), also known as membrane cofactor protein due to its involvement in complement activation, which is used by measles vaccine strains for cellular entry (18-20). Notably, both malignant and non-malignant cells among the PBMCs 

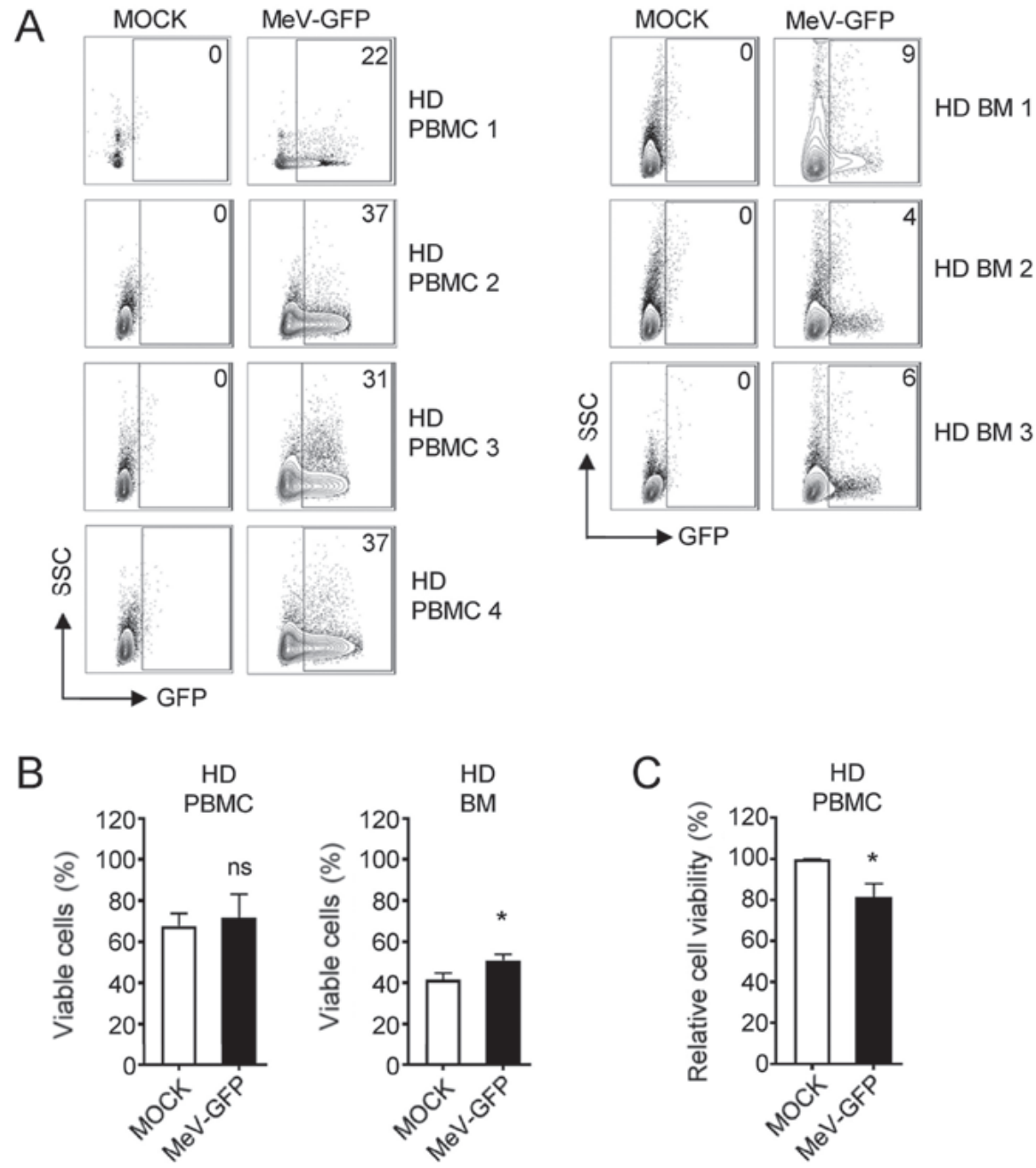

Figure 3. MeV does not exert tumoricidal effects against cells of HD. PBMCs or BM cells of HD were infected with MeV-GFP or MOCK-infected and analyzed at 4 dpi. (A) GFP expression of the indicated cells was investigated by FACS. The percentage of positive cells is depicted. (B) Cell number was determined using the Countess Automated Cell Counter. The percentage of living cells is indicated. HD PBMC ( $=4)$, HD BM ( $n=3)$. (C) Viability of cells was measured using CellTiter-Blue viability assay. $\operatorname{HD}$ PBMC $(\mathrm{n}=4)$. Statistically significant differences $(\mathrm{P}<0.05)$ are indicated by an asterisk $(*)$; ns, not significant. $\mathrm{MeV}$, measles vaccine virus; PBMCs, peripheral blood mononuclear cells; HD, healthy donor; BM, bone marrow.

of patients with AML displayed a profound expression of CD46 (Fig. 4B).

Viral infections are known to induce IFN-stimulated genes via the activation of cytoplasmic receptors, such as RIG-I or MDA5. In tumor cells, this pathway often displays mutations, thus rendering cells more susceptible to virotherapy (3). Thus, in this study, we then examined the modulation of IFN signaling in AML cell lines upon parallel infection with MeV-GFP. For this purpose, the cells were lysed at the indicated time points post-infection and the phosphorylation of STAT1 (phospho-STAT1) and the expression of the downstream prototypic interferon-stimulated gene, IFIT-1, was examined by western blot analysis (Fig. 4C). STAT1 was found to be phosphorylated upon the infection of AML cell lines with MeV-GFP during the time course studied (24, 48 and $72 \mathrm{hpi}$ ), while only minimal levels of phospho-STAT1 were detectable in the MOCK-infected cells. While phospho-STAT1 was expressed most profoundly at 24 hpi in the MM- 6 cells, highest levels in NOMO-1 and SKM-1 cells were observed at $48 \mathrm{hpi}$. This is in line with our results obtained with the time course analysis of $\mathrm{MeV}$ infection rates, where the MM-6 cells exhibited rapidly highest infection levels as compared to the other cell lines (Fig. S1). A comparable kinetic was observed with regard to IFIT-1 expression, which was again specifically induced upon $\mathrm{MeV}$ infection; detectable levels peaked between 24 hpi (MM-6 cells) and 48-72 hpi (NOMO-1 and SKM-1 cells). In MM- 6 cells only trace amounts of IFIT-1 could be detected at 48 and 72 hpi. Notably, the activation of IFN signaling upon $\mathrm{MeV}$ infection was also observed in samples derived from patients with AML, as determined by the analysis of phospho-STAT1 and IFIT-1 expression (Fig. 4D). Again, only minor levels of phospho-STAT1 were observed in the MOCK-infected cells, whereas $\mathrm{MeV}$ infection led to a substantial increase in the levels of phospho-STAT1. By contrast, IFIT-1 expression was only induced upon infection with MeV-GFP. Herein, protein expression appeared to vary moderately during the analyzed time course. Highest levels of phospho-STAT1, as well as IFIT-1 in PBMCs from patients with AML were detectable at approximately $72 \mathrm{hpi}$. In line with this, AML cells were found to release IFN- $\beta$ at $48 \mathrm{hpi}$, which preceded the maximum levels of the interferon-induced proteins detected by western blot analysis (Fig. S3). Taken 
A

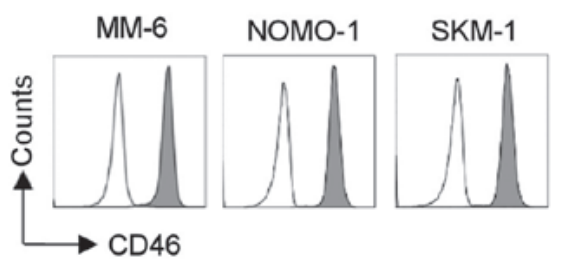

B
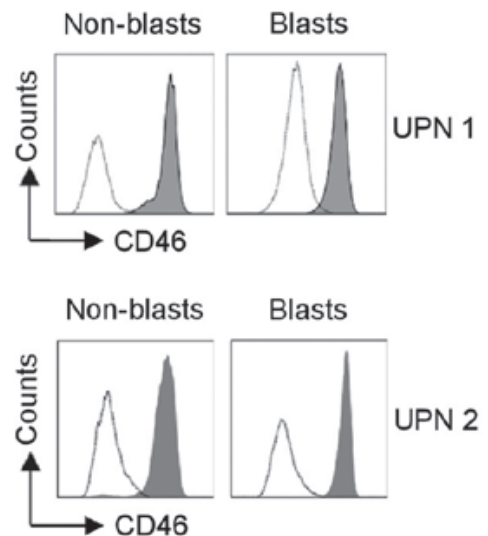

C

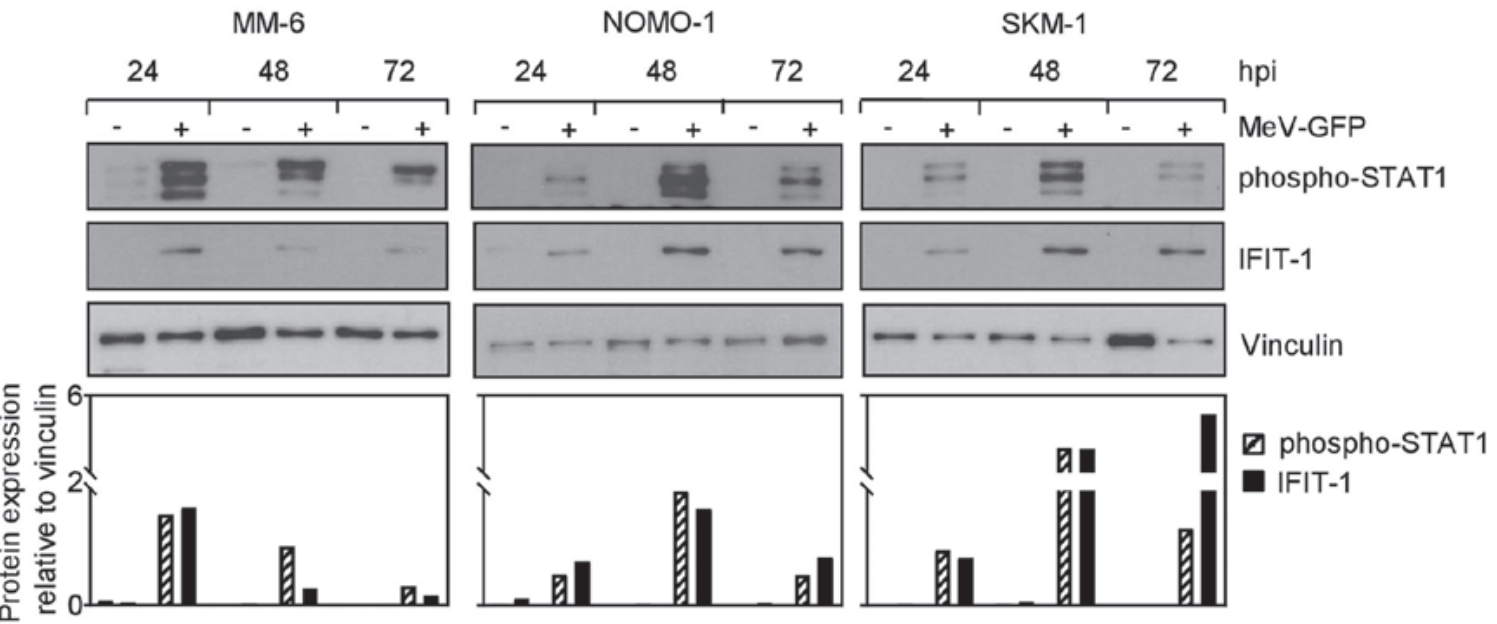

D

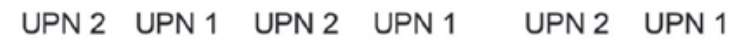

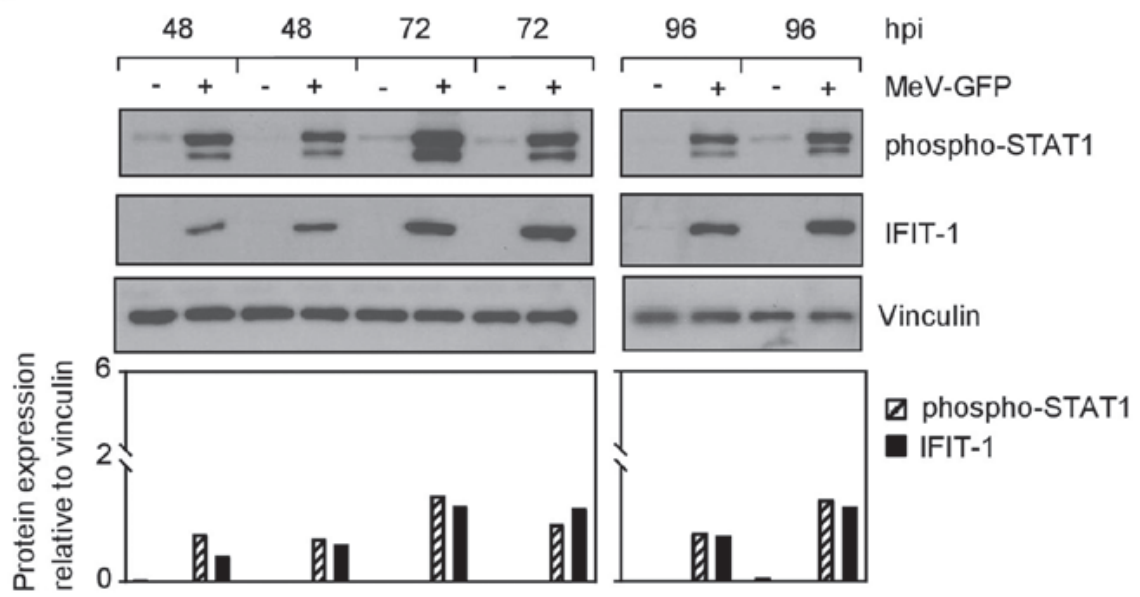

Figure 4. AML cells express MeV entry receptor CD46 and induce IFN signaling upon MeV infection. (A and B) CD46 surface expression on (A) AML cell lines and (B) PBMCs from patients with AML was investigated by FACS. Shaded peaks, anti-CD46; open peaks, isotype control. (C) AML cell lines and (D) PBMCs from patients with AML were infected with MeV-GFP or MOCK-infected. Cells were lysed at the indicated time points. Subsequently, the phosphorylation of STAT1 and the expression of IFIT1 were examined by western blot analysis (top panels) and semi-quantified (lower panels). Vinculin served as loading control. (A-D) One representative result out of 2 experiments with similar results is shown. AML, acute myeloid leukemia; MeV, measles vaccine virus; IFN, interferon; PBMCs, peripheral blood mononuclear cells; STAT1, signal transducer and activator of transcription 1; IFIT1, interferon-induced protein with tetratricopeptide repeats 1 .

together, these findings unravel induction of IFN signaling in AML cells upon infection with oncolytic MeV.

Administration of the prodrug, 5-FC, increases the MeV-mediated lysis of AML cells. To investigate whether targeted, i.e., tumor cell-restricted chemotherapy can further improve the lysis of $\mathrm{MeV}$-targeted AML cells, we employed $\mathrm{MeV}-\mathrm{SCD}$, a variant $\mathrm{MeV}$ vector which is 'armed' with super cytosine deaminase that enables conversion of the prodrug 5-FC into the active compound, 5-FU. 

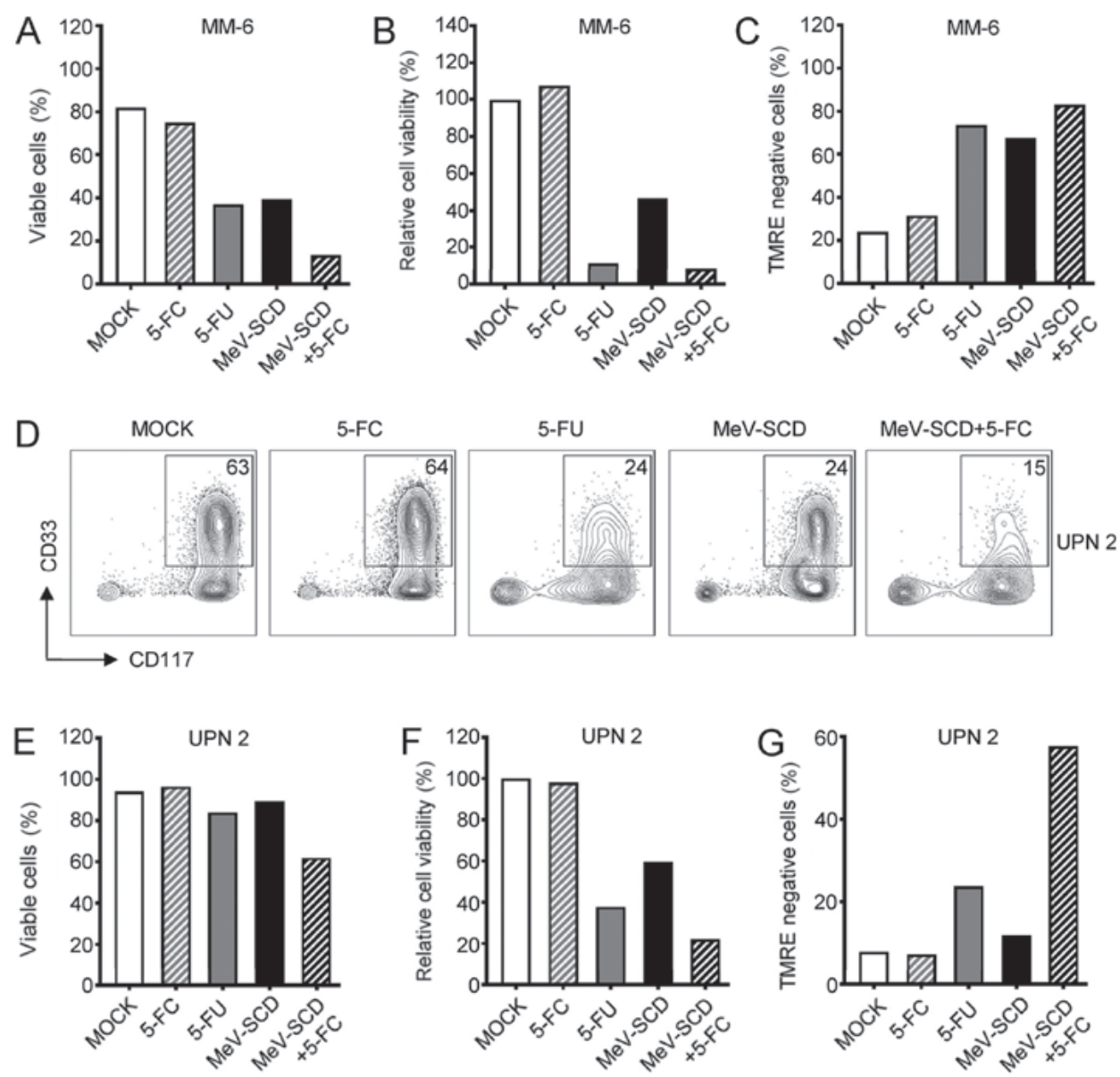

Figure 5. AML cells can be targeted by a combination of MeV-SCD and 5-FC. (A-C) MM-6 cells or (D-G) the PBMC fraction from a patient with AML (UPN 2) were infected with MeV-SCD or MOCK-infected. Cells were analyzed at 4 dpi. (A and E) Cell number was determined using the Countess Automated Cell Counter. The percentage of living cells is indicated (MM-6 and UPN 2, $\mathrm{n}=2$ each). (B and F) Viability of cells was measured using CellTiter-Blue viability assay. MM-6 ( $\mathrm{n}=2)$; UPN $2(\mathrm{n}=1)$. (C and G) Apoptosis was determined by staining with TMRE and subsequent FACS analysis. The percentage of TMRE-negative cells is indicated. MM-6 ( $\mathrm{n}=2)$; UPN $2(\mathrm{n}=1)$. (D) The percentage of leukemic blasts is displayed. (A-C and E-G) Values depict means of data from the indicated number of independent experiments. AML, acute myeloid leukemia; MeV, measles vaccine virus; PBMC, peripheral blood mononuclear cell; UPN, unique patient number.

Direct oncolysis by $\mathrm{MeV}-\mathrm{SCD}$ in the absence of the prodrug was comparable with MeV-GFP (data not shown). While 5-FC did not alter the percentage of viable cells, the viability or the apoptosis of MM-6 cells, the presence of equimolar amounts of 5-FU substantially decreased the parameters of cell viability and induced apoptosis, as determined by TMRE negativity (Fig. 5A-C). Notably, the direct effects of MeV-SCD were comparable to those of 5-FU with regard to cell viability and apoptosis in the experimental setting with MM-6 cells. However, the combined use of MeV-SCD and the prodrug, 5-FC, profoundly diminished the percentage of viable cells with MeV-SCD or 5-FU as compared to monotherapy. Since 5-FU almost completely blocked metabolic activity in this experiment, no further decrease could be observed upon the combined administration of MeV-SCD and 5-FC. In line with this, as the active compound 5-FU already induced high rates of apoptosis of the MM-6 cells, the combined use of the prodrug and MeV-SCD only slightly increased the apoptotic rates as compared to monotherapy with 5-FU alone.

Subsequently, we investigated whether the combined application of MeV-SCD and the prodrug, 5-FC, enhances the lysis of primary AML cells from patients. FACS analysis was employed to monitor the modulation of the percentage of leukemic blasts among the PBMCs. The presence of the prodrug alone did not alter the percentage of $\mathrm{CD} 33^{+} / \mathrm{CD} 117^{+}$ leukemic blasts of UPN 2, although the percentage of blasts was clearly reduced in the presence of 5-FU (Fig. 5D). Again, MeV-SCD-mediated leukemia cell lysis was comparable to the effects obtained with 5-FU in this setting. The effects observed with the combined use of MeV-SCD and the prodrug, 5-FC, however, were superior to those mediated by either MeV-SCD or the active compound 5-FU alone. These data were confirmed and were extended by the analyses of PBMCs from patients with AML with regard to the percentage of viable cells (Fig. 5E), cell viability (Fig. 5F) and apoptotic rates as determined by TMRE negativity (Fig. 5G). While these parameters were not altered in the presence of the prodrug, 5-FU led to a substantial decrease in cell viability which was paralleled by apoptosis. The application of MeV-SCD led to a less pronounced downmodulation of cell viability and increase of TMRE negative cell count as compared to 5-FU. Combination therapy using MeV-SCD together with 5-FC surpassed the effects obtained with either $\mathrm{MeV}$ monotherapy or administration of 5-FU, both with regard to cell viability and apoptosis. 
In summary, the application of the prodrug, 5-FC, together with the suicide gene-enhanced oncolytic virus MeV-SCD constitutes a novel therapeutic regimen which clearly enhances the MeV-mediated oncolysis in the treatment of AML.

\section{Discussion}

The therapy of AML remains a major challenge in clinical hematology with a highly unmet medical need, underlining the necessity for improvements of presently available treatment modalities. Oncolytic viruses have shown therapeutic success in various tumor entities, including hematological malignancies (6). Highly promising responses have been obtained in preclinical and clinical studies where virotherapy was used in combination with chemotherapeutic or immunotherapeutic agents (21-26).

In this study, we investigated the combined use of measles vaccine virus and the tumor site-specific generation of 5-FU for the treatment of AML. We took advantage of the measles vaccine virus $\mathrm{MeV}$ (MeV-GFP) and its engineered variant MeV-SCD (27), the latter facilitating the conversion of the prodrug, 5-FC, into the therapeutically active compound, 5-FU, and employed both $\mathrm{MeV}$ derivatives for the infection of AML cell lines and primary cells from patients with AML.

We found that all employed AML cell lines displayed substantial $\mathrm{MeV}$ infection rates of at least 59\%, which was paralleled by a reduction of the percentage of viable cells and metabolic activity, as well as the induction of apoptosis. The reduction of cell viability quantitatively excelled differences in the percentage of viable cells, which does not account for the loss of dead cells by the generation of cellular debris upon $\mathrm{MeV}$ mediated oncolysis, and was associated with the amount of TMRE-negative cells. Infection rates per se did not reflect the tumoricidal effect obtained upon $\mathrm{MeV}$ infection, since not all infected cells were found to be susceptible to direct oncolysis. Notably, leukemic blasts among PBMCs from patients with AML appeared to be preferentially lysed by $\mathrm{MeV}$ suggesting its usage as therapeutic strategy. In stark contrast to conventional treatment regimens, this virotherapy is believed to affect non-malignant cells to a much lesser degree, particularly those of the BM, and thus allows for hematopoiesis to be sustained post-treatment. However, while the direct oncolysis of primary cells was less pronounced in the ex vivo setting as compared to AML cell lines, the metabolic activity of primary cells was profoundly reduced. Notably, the infection of non-malignant patient cells was relatively precluded although the surface expression of CD46, the entry receptor for $\mathrm{MeV}$ vaccine strains (18), was found on both, non-malignant, as well as leukemic cells among PBMCs from patients with AML. Yet, leukemic blasts which were preferentially susceptible to $\mathrm{MeV}$ infection in our study displayed comparable or even reduced surface levels of CD46, which suggests the presence of other factors for this oncotropism. Of note, no essential decrease with regard to cell count was observed with PBMCs and BM cells from healthy donors. One may speculate that this oncotropism is due to alterations in the cellular IFN machinery in transformed cells. In this respect, the type I IFN family acts as early response to viral infections creating an antiviral state within the host cell and hitherto uninfected neighboring cells (10). AML cells were found to release IFN- $\beta$, resulting in the activation of the
JAK/STAT pathway, as determined by the phosphorylation of STAT1 and the downstream induction of the interferon stimulated gene, IFIT1, upon MeV infection. In line with this, MM- 6 cells which were most susceptible to $\mathrm{MeV}$ infection and direct oncolysis, exhibited the weakest activation of the IFN signaling pathway, resulting in only a minimal expression of IFIT-1 post MeV infection. These observations may suggest the consideration of additionally targeting the IFN pathway in order to enhance response towards MeV-based virotherapy.

To improve efficacy, we set out to combine direct oncolysis with tumor site specific generation of chemotherapy. For this purpose, we employed a $\mathrm{MeV}$ variant armed with super cytosine deaminase (MeV-SCD) $(3,12)$, which catalyzes conversion of the prodrug, 5-FC, into the active and clinically approved compound, 5-FU, which acts via the inhibition of thymidylate synthase and incorporation of its metabolites into nucleic acids (14). While 5-FC did not alter cell viability or the apoptosis rates, 5-FU exerted profound cytotoxic effects in most settings, and accordingly, the systemic administration thereof does not constitute a promising therapeutic strategy for treatment of AML. MeV-SCD facilitated direct oncolysis in the absence of the prodrug, but was not able to eliminate AML cells in the in vitro conditions employed in this study. However, the addition of 5-FC enhanced the tumoricidal effects of MeV-SCD in all cases. Remarkably, this was also the case when leukemic cells displayed only moderate susceptibility to the oncolytic virus and hence were not affected by direct oncolysis.

With regard to the clinical development of suicide gene-based approaches, two open and three completed clinical trials used vocimagene amiretrorepvec (Toca 511) for the treatment of glioblastoma. This gamma-retroviral replicating vector encodes cytosine deaminase and exploits the strategy to tumor-selectively produce active $5-\mathrm{FU}(28,29)$. Thus far, these trials have revealed both anticancer activity and a favorable safety profile. Albeit the strategy to target thymidylate synthase constitutes also a promising option for the treatment of AML, 5-FU rarely has been used clinically due to i) overlapping gastrointestinal and hematological toxicities with standard anti-leukemic drugs; and even more due to ii) its limited potency as compared to its toxic effects. The latter are especially detrimental as they also affect stem cells (30). This is of utmost importance, since we did not see infection or considerable alteration of the viability of BM cells upon $\mathrm{MeV}$ infection, which would precede the site-specific generation of the chemotherapeutic agent, 5-FU, in the stem cell niche. To elucidate the underlying mechanisms of this inherent resistance of BM cells is subject of an ongoing study. Our approach to locally generate the active compound in a leukemia-targeted manner prevents systemically relevant levels of 5-FU and thus may prohibit the fatal toxicities to be expected upon combinatorial systemic administration of 5-FU in AML.

Of note, the data of this manuscript has in part been presented as a poster at the Annual Meeting of the German, Austrian and Swiss Societies for Hematology and Oncology, DGHO, September, 29 to October 03, 2017, Stuttgart, Germany and the abstract has been previously published (31).

In conclusion, in this study, for the first time, to the best of our knowledge, we preclinically evaluated the combinatorial use of measles vaccine virus and 5-FU for the treatment of AML. Our approach to tumor-selectively produce the active drug and to combine its effects with direct lytic effects of 
virotherapy may overcome present limitations and constitutes a promising strategy to introduce 5-FU in the treatment of AML.

\section{Acknowledgements}

Flow cytometry sample acquisition was in part performed on shared instruments of the Flow Cytometry Core Facility Tuebingen.

\section{Funding}

This study was supported by DFG (SA1360/7-3), Deutsche Krebshilfe $(111828,111134)$ and Germany's Excellence Strategy (EXC 2180/1) (to HRS). This study was also supported by the Institutional Strategy of the University of Tübingen (Deutsche Forschungsgemeinschaft, ZUK 63) (to SM).

\section{Availability of data and materials}

The datasets used and/or analyzed during the current study are available from the corresponding author on reasonable request.

\section{Authors' contributions}

SM performed and interpreted the FACS experiments, and prepared the patient samples. SB performed the virus infection experiments, western blot analysis and the analyses of cell viability. IS performed the ELISA tests. UML and HRS conceived and designed the study. All authors contributed to the writing of the manuscript; all authors have read and approved the final manuscript.

\section{Ethics approval and consent to participate}

This study was approved by the Ethics Committee at the Medical Faculty of the Eberhard Karls University and the University Hospital Tuebingen (reference no. 13/2007V). Informed consent was obtained from all patients and healthy donors in accordance with the Helsinki protocol.

\section{Patient consent for publication}

Not applicable.

\section{Competing interests}

All authors declare that they have no competing interests.

\section{References}

1. Wattad M, Weber D, Döhner K, Krauter J, Gaidzik VI, Paschka P, Heuser M, Thol F, Kindler T, Lübbert M, et al: Impact of salvage regimens on response and overall survival in acute myeloid leukemia with induction failure. Leukemia 31: 1306-1313, 2017.

2. Kelly E and Russell SJ: History of oncolytic viruses: Genesis to genetic engineering. Mol Ther 15: 651-659, 2007.

3. Berchtold S, Lampe J, Weiland T, Smirnow I, Schleicher S, Handgretinger R, Kopp HG, Reiser J, Stubenrauch F, Mayer $\mathrm{N}$, et al: Innate immune defense defines susceptibility of sarcoma cells to measles vaccine virus-based oncolysis J Virol 87: 3484-3501, 2013.

4. Russell SJ, Peng KW and Bell JC: Oncolytic virotherapy. Nat Biotechnol 30: 658-670, 2012.
5. Cattaneo R, Miest T, Shashkova EV and Barry MA: Reprogrammed viruses as cancer therapeutics: Targeted, armed and shielded. Nat Rev Microbiol 6: 529-540, 2008.

6. Aref S, Bailey K and Fielding A: Measles to the rescue: A rview of oncolytic measles virus. Viruses 8: 8, 2016.

7. Takeuchi $\mathrm{O}$ and Akira S: MDA5/RIG-I and virus recognition. Curr Opin Immunol 20: 17-22, 2008.

8. Hornung V,Ellegast J,Kim S, Brzózka K, Jung A,Kato H,Poeck H, Akira S, Conzelmann KK, Schlee M, et al: 5'-Triphosphate RNA is the ligand for RIG-I. Science 314: 994-997, 2006.

9. Darnell JE Jr, Kerr IM and Stark GR: Jak-STAT pathways and transcriptional activation in response to IFNs and other extracellular signaling proteins. Science 264: 1415-1421, 1994.

10. Berghäll H, Sirén J, Sarkar D, Julkunen I, Fisher PB, Vainionpää R and Matikainen S: The interferon-inducible RNA helicase, mda-5, is involved in measles virus-induced expression of antiviral cytokines. Microbes Infect 8: 2138-2144, 2006.

11. Childs K, Stock N, Ross C, Andrejeva J, Hilton L, Skinner M, Randall R and Goodbourn S: mda-5, but not RIG-I, is a common target for paramyxovirus V proteins. Virology 359: 190-200, 2007.

12. Graepler F, Lemken ML, Wybranietz WA, Schmidt U, Smirnow I, Gross CD, Spiegel M, Schenk A, Graf H, Lauer UA, et al: Bifunctional chimeric SuperCD suicide gene -YCD: YUPRT fusion is highly effective in a rat hepatoma model. World $\mathrm{J}$ Gastroenterol 11: 6910-6919, 2005.

13. Pol J, Vacchelli E, Aranda F, Castoldi F, Eggermont A, Cremer I, Sautès-Fridman C, Fucikova J, Galon J, Spisek R, et al: Trial Watch: Immunogenic cell death inducers for anticancer chemotherapy. OncoImmunology 4: e1008866, 2015.

14. Longley DB, Harkin DP and Johnston PG: 5-fluorouracil: Mechanisms of action and clinical strategies. Nat Rev Cancer 3: 330-338, 2003.

15. Zhang LF, Tan DQ, Jeyasekharan AD, Hsieh WS, Ho AS, Ichiyama K, Ye M, Pang B, Ohba K, Liu X, et al: Combination of vaccine-strain measles and mumps virus synergistically kills a wide range of human hematological cancer cells: Special focus on acute myeloid leukemia. Cancer Lett 354: 272-280, 2014.

16. Kaerber G: Beitrag zur kollektiven Behandlung pharmakologischer Reihenversuche. Naunyn-Schmiedeberg's Arch. Pharmacol 162: 480-483, 1931 (In German).

17. Spearman C: The method of 'right and wrong cases' ('constant stimuli') without Gauss's formulae. Br J Psychol 2: 227-242, 1908.

18. Hashiguchi T, Maenaka K and Yanagi Y: Measles virus hemagglutinin: Structural insights into cell entry and measles vaccine. Front Microbiol 2: 247, 2011.

19. Naniche D, Varior-Krishnan G, Cervoni F, Wild TF, Rossi B, Rabourdin-Combe $\mathrm{C}$ and Gerlier D: Human membrane cofactor protein (CD46) acts as a cellular receptor for measles virus. J Virol 67: 6025-6032, 1993.

20. Dörig RE, Marcil A, Chopra A and Richardson CD: The human CD46 molecule is a receptor for measles virus (Edmonston strain). Cell 75: 295-305, 1993.

21. Ribas A, Dummer R, Puzanov I, VanderWalde A, Andtbacka RHI, Michielin O, Olszanski AJ, Malvehy J, Cebon J, Fernandez E, et al: Oncolytic virotherapy promotes intratumoral $\mathrm{T}$ cell infiltration and improves anti-PD-1 immunotherapy. Cell 170: 1109-1119 e1110, 2017.

22. Liu Z, Ravindranathan R, Kalinski P, Guo ZS and Bartlett DL: Rational combination of oncolytic vaccinia virus and PD-L1 blockade works synergistically to enhance therapeutic efficacy. Nat Commun 8: 14754, 2017.

23. Cervera-Carrascon V, Siurala M, Santos JM, Havunen R, Tähtinen S, Karell P, Sorsa S, Kanerva A and Hemminki A: TNF $\alpha$ and IL-2 armed adenoviruses enable complete responses by anti-PD-1 checkpoint blockade. OncoImmunology 7: e1412902, 2018.

24. Binz E and Lauer UM: Chemovirotherapy: Combining chemotherapeutic treatment with oncolytic virotherapy. Oncolytic Virother 4: 39-48, 2015.

25. Binz E, Berchtold S, Beil J, Schell M, Geisler C, Smirnow I and Lauer UM: Chemovirotherapy of pancreatic adenocarcinoma by combining oncolytic vaccinia virus GLV-1h68 with $n a b$-paclitaxel plus gemcitabine. Mol Ther Oncolytics 6: 10-21, 2017.

26. Mahalingam D, Goel S, Aparo S, Patel Arora S, Noronha N, Tran H, Chakrabarty R, Selvaggi G, Gutierrez A, Coffey M, et al: A phase II study of pelareorep (REOLYSIN ${ }^{\circledR}$ ) in combination with gemcitabine for patients with advanced pancreatic adenocarcinoma. Cancers (Basel) 10: 10, 2018. 
27. Noll M, Berchtold S, Lampe J, Malek NP, Bitzer M and Lauer UM: Primary resistance phenomena to oncolytic measles vaccine viruses. Int J Oncol 43: 103-112, 2013.

28. Pol J, Buqué A, Aranda F, Bloy N, Cremer I, Eggermont A, Erbs P, Fucikova J, Galon J, Limacher JM, et al: Trial Watch-Oncolytic viruses and cancer therapy. OncoImmunology 5: e1117740, 2015.

29. Cloughesy TF, Landolfi J, Vogelbaum MA, Ostertag D, Elder JB, Bloomfield S, Carter B, Chen CC, Kalkanis SN, Kesari S, et al: Durable complete responses in some recurrent high-grade glioma patients treated with Toca 511 + Toca FC. Neuro-oncol 20 1383-1392, 2018.

30. Pardee TS, Gomes E, Jennings-Gee J, Caudell D and Gmeiner WH: Unique dual targeting of thymidylate synthase and topoisomerase1 by FdUMP[10] results in high efficacy against AML and low toxicity. Blood 119: 3561-3570, 2012.
31. der Deutschen J: Suicide gene-armed measles vaccine virus for the treatment of AML. In: Proceedings of the Annual Meeting of the German Society for Haematology and Medical Oncology (DGHO) in Stuttgart. Oncol Res Treat 40 (Suppl 3): 1-308, 2017.

This work is licensed under a Creative Commons Attribution-NonCommercial-NoDerivatives 4.0 International (CC BY-NC-ND 4.0) License. 